\title{
Anal human papillomavirus infection and its relations with abnormal anal cytology among MSM with or without HIV infection in Japan
}

Daisuke Shiojiri ( $\nabla$ dshiojiri@hosp.ncgm.go.jp )

National Center For Global Health and Medicine

Daisuke Mizushima

National Center For Global Health and Medicine

Misao Takano

National Center For Global Health and Medicine

Koji Watanabe

National Center For Global Health and Medicine

Naokatsu Ando

National Center For Global Health and Medicine

Haruka Uemura

National Center For Global Health and Medicine

Yasuaki Yanagawa

National Center For Global Health and Medicine

Takahiro Aoki

National Center For Global Health and Medicine

Junko Tanuma

National Center For Global Health and Medicine

Kunihisa Tsukada

National Center For Global Health and Medicine

Katsuji Teruya

National Center For Global Health and Medicine

Yoshimi Kikuchi

National Center For Global Health and Medicine

Hiroyuki Gatanaga

National Center For Global Health and Medicine

Shinichi Oka

National Center For Global Health and Medicine 
Keywords: human papillomavirus, high risk genotype, pre-cancer, men having sex with men, HIV, 9 valent HPV vaccine

Posted Date: February 24th, 2021

DOI: https://doi.org/10.21203/rs.3.rs-232653/v1

License: (c) (1) This work is licensed under a Creative Commons Attribution 4.0 International License. Read Full License 


\section{Abstract}

Background Anorectal high-risk human papillomavirus (hr-HPV) infection is associated with anal malignancies. However, the epidemiological data is quite limited in Japan.

Methods Anorectal swab was obtained from HIV-infected and - uninfected men who have sex with men (MSM). Only high-risk HPV genotypes were examined by the Hybrid Capture-based methodology. Anal cytology was determined by the modification of the Bethesda System classification.

Results In total, 644 participants were included. No participant had history of HPV vaccination at inclusion. The overall prevalence of hr-HPV was 58.5\% (95\% confidence interval: 54.7-62.3). The most frequently detected hr-HPV genotypes in order were HPV-52,-16, and -58. Among the HPV infected subjects, $82.8 \%(312 / 377)$ were infected with at least one of the 9 valent vaccine covered genotypes. Incidence of abnormal cytology was positively correlated with the number of HPV genotypes infected rather than the difference in genotypes. Multivariate analysis identified the number of detected hr-HPV genotypes as well as HIV-infection were the independent risk factors for pre-cancer.

Conclusion Nine valent vaccine preventable HPVs are currently prevalent among MSM in Japan. Also, increased numbers of hr-HPV genotypes were strongly associated with anorectal pre-cancer. Universal HPV vaccination should be considered for Japanese MSM.

\section{Introduction}

Anorectal chronic infection of high-risk types of human papillomavirus (hr-HPV) and related malignancies are the growing concern of public health among men who have sex with men (MSM). Prevalence of hrHPV is extremely high not only among the cases of anal cancer but also among asymptomatic MSM ${ }^{1,2}$. Also, the positivity of hr-HPV and the risk of anorectal cancer are even greater in HIV-infected individuals ${ }^{3,4,5}$. Recently, routine anal cancer screening using anal cytology, is recommended in many of developed countries ${ }^{6}$. Also, HPV vaccination is recommended for all male and female adolescents (from 9 through 26 years) in such countries. Moreover, in 2019, Advisory Committee on Immunization Practices (ACIPs) has recommended a shared clinical decision-making regarding HPV vaccination for some adults aged 27 through 45 years who are not adequately vaccinated ${ }^{7,8}$, although preventive effects of vaccination on anal cancer or newly acquisition of hr-HPV in sexually active aged adult population have not been clearly demonstrated in previous studies.

On the other hand, in Japan, although HPV vaccine for male individuals was approved only very recently, genotyping of anorectal hr-HPV and anal-canal cancer screening are not currently approved. Furthermore, epidemiological data of MSM, which includes the prevalent hr-HPV genotypes and the related histopathological abnormalities, are quite limited, although previously reported preliminary data suggested its high prevalence ${ }^{9}$. 
In the present study, we assessed the epidemiology of anorectal colonization of HPV and related abnormal cytology among MSM and sought to discuss the future strategy for preventing HPV related cancer in Japan.

\section{Results}

\section{Characteristics of the study participants}

We enrolled a total of 644 MSM that included 437 HIV-infected and 207 HIV-uninfected eligible consenting participants. The demographic characteristics according to HIV-infected and uninfected subjects are presented in Table 1. HIV-infected participants were 10 years older compared with HIVuninfected. All subjects reported having anal intercourse with other men. No participants had past history of HPV vaccination at inclusion. Compared to HIV-uninfected, infected subjects had significantly higher number of sexual partners in lifetime, but with less sexual partners in past 6 months than uninfected subjects. No significant difference in condom usage during anal sex in both groups, while history of STI was significantly higher in HIV-infected than uninfected, most acquiring syphilis followed by anal warts, gonorrhea, chlamydia and herpes. All HIV-infected individuals were on treatment by anti-retroviral therapy (ART). Plasma HIV-RNA were well-suppressed, and CD4 counts were high. 
Table 1

Demographic characteristics of HIV-infected and HIV-uninfected Japanese MSM

\begin{tabular}{|c|c|c|c|}
\hline & $\begin{array}{l}\text { HIV-infected } N=437 \\
N(\%), \text { or median (IQR) }\end{array}$ & $\begin{array}{l}\text { HIV-uninfected N = } 207 \\
\text { N (\%), or median (IQR) }\end{array}$ & $P$ value \\
\hline Age, years & $47.0(38.9-56.5)$ & $37.3(29.2-46.5)$ & $<.001$ \\
\hline No. of sex partner in lifetime & $184.0(47.2-794.6)$ & $13.6(4.4-36.3)$ & $<.001$ \\
\hline $\begin{array}{l}\text { No. of sex partner in the past } 6 \\
\text { months }\end{array}$ & $4.6(1.1-13.8)$ & $9.2(1.9-27.8)$ & $<.001$ \\
\hline$\%$ use of condom & $57.8(27.5-89.0)$ & $61.5(23.2-88.8)$ & .256 \\
\hline Past history of STIs & $317(72.5)$ & $72(34.8)$ & $<.001$ \\
\hline Syphilis & $222(50.8)$ & $54(26.1)$ & $<.001$ \\
\hline Condyloma (anal warts) & $90(20.6)$ & $2(1.0)$ & $<.001$ \\
\hline Gonorrhea & $69(15.8)$ & $7(3.4)$ & $<.001$ \\
\hline Chlamydia & $63(14.4)$ & $14(6.8)$ & .006 \\
\hline Herpes & $43(9.8)$ & $2(1.0)$ & $<.001$ \\
\hline Latest CD $4+\mathrm{T}$-cell count $\times 10^{9} / \mathrm{L}(\mathrm{IQR})$ & $687.6(0-12.3)$ & NA & NA \\
\hline HIV-RNA copies/mL & UD (UD-<20) & NA & NA \\
\hline Any hr-HPV positive & $293(67.0)$ & $84(40.6)$ & $<.001$ \\
\hline
\end{tabular}

\section{Prevalence of human papilloma virus}

Overall positive rate of hr-HPV was 58.5\% [95\% confidence interval: 54.7-62.3]. HIV-infected MSM (67.0\% [62.5-71.3]) had significantly higher infection rate as compared to HIV-uninfected (40.6\% [34.1-47.4]) (Table 1). As demonstrated in Fig. 1, more than half of hr-HPV infected individuals (211/377) possessed multiple hr-HPV genotypes. Of note, the number of hr-HPV genotypes infected were higher in HIV-infected subjects. The positivity of each hr-HPV genotype to study participants was shown in Supplementary Fig S1 online. HPV genotypes 16, 52, and 58 were most frequently detected. Also, we confirmed that most of HPV-infected subjects $(312 / 377)$ possessed at least one of the 9 valent vaccine covered genotypes (genotype 16, 18, 31, 33, 45, 52, and 58). All hr-HPV genotypes other than genotype 59 were more frequently seen among HIV-infected MSM, although only genotype 52 showed statistical significance (See Supplementary Table S1 online). Most of genotypes were prevalent equally in different age group (See Supplementary Fig S2 online). However, GT 31 was more frequently seen among younger population (30 y.o. or younger), whereas GT $52 \& 56$ were more frequently seen among fifties. Considered together, 
MSM in Japan, especially HIV-infected, are highly affected by hr-HPV, moreover, 9 valent HPV vaccine covered genotypes are commonly seen among them.

\section{Prevalence of abnormal anal cytology}

Anal cytology results in accordance with Bethesda classification are presented in Supplementary Fig S3 online. Abnormal cytology results were seen in 46 cases (10.5\% [86.2-92.0]) of 437 HIV-infected individuals, which consists of 18 cases of LSIL, 16 cases of ASC-H, and 12 cases of HSIL. On the other hand, only 2 cases (1.0\% [0.2-3.5]) of 207 HIV-uninfected had abnormal cytology results (both were ASCH). Next, in order to assess the impact of HPV genotypes on abnormal cytology result, frequencies of abnormal cytology results in accordance with hr-HPV infection were calculated. Frequency of abnormal cytology among hr-HPV positive individuals was 11.4\% [8.6-15.0], whereas those among hr-HPV negative participants was $1.9 \%$ [0.8-4.3] (Fig. 2a). Importantly, the frequency of abnormal cytology was not different among different genotypes (Fig. 2a), however, it was positively correlated with the number of HPV genotypes detected (Fig. 2b). From these results, abnormal cytology was more frequently seen among HIV-infected individuals than those among HIV-uninfected. Furthermore, incidence of anorectal pre-cancer could be more influenced by the number of hr-HPV genotypes than the difference in its genotype.

\section{Risk factors for Pre-cancer}

Finally, in order to identify the affecting factors on the incidence of pre-cancer, uni- and multi-variate regression analyses were performed. In univariate analysis, age, HIV infection and number of detected hrHPV genotypes were significantly associated with abnormal cytology results, as shown odds ratio of $1.03,12.06$ and 1.65 , respectively. As expected, in the multivariate analysis, HIV infection and number of $\mathrm{hr}-\mathrm{HPV}$ genotypes detected were identified as independent risk factors for pre-cancer. Especially, number of hr-HPV genotypes were the very strong one (Odds Ration 1.57, P value <.001) (See Supplementary Table S1).

\section{Discussion}

In our study, the prevalence of anal hr-HPV infection among MSM cohort was 58.5\% [54.7-62.3], and the most frequently detected hr-HPV genotypes in order were HPV-52 (18.5\%), HPV-16 (16.9\%), and HPV-58 (15.7\%) among them. Our previous study conducted 10 years ago in the HIV-infected MSM cohort identified prevalence of hr-HPV infection as high as $75.9 \%$, and the most frequent hr-HPV genotypes in order were HPV-58 (30.2\%), HPV-16 (28.8\%) and HPV-52 (22.2\%) ${ }^{9}$. Considered together, these 3 genotypes are predominant hr-HPV genotypes among Japanese MSM throughout in this decade. Our study also revealed similar prevalence among HIV-infected subjects 67.0\% [62.5-71.3], while HIV-uninfected subjects had a lower rate of $40.6 \%$ [34.1-47.4].The high prevalence with hr-HPV in MSM are almost concurrent with those reported in other Asian countries, but the distribution of hr-HPV genotypes was somewhat different ${ }^{4,14,15}$. The prevalence of HPV infection was $85.2 \%$ in Taiwan, $82.7 \%$ in China and $85 \%$ 
in Thailand, while most frequent HPV genotypes were HPV-16, HPV-51 and HPV-52 in Taiwan and Thailand, HPV-16 and HPV-18 in China ${ }^{4,14,15}$.

All of 9 valent HPV vaccine covered genotypes (genotypes 16, 18, 31, 33, 45, 52 and 58 are high-risk HPV genotypes, while genotypes 6 and 11 are low-risk HPV genotypes) were not rarely (> 5\%) identified in the study participants (See supplementary Fig S1 online). Moreover, most of HPV infected subjects (312/377) possessed at least one of the 9 valent vaccine covered genotypes. These results indicate that vaccine preventable genotypes are currently common hr-HPV among Japanese MSM.

Even more interestingly, it was clearly documented for the first time in Japan in the present study that the incidence of abnormal cytology was positively correlated with the number of HPV genotypes detected (Fig. 2b, Table 2), as was discussed in a previous study ${ }^{14}$. On the other hand, the incidence of abnormal cytology was not greatly influenced by the difference in genotypes (Fig. 2a). Considered together, HPV vaccination could be recommended even for those who were already infected with some hr-HPV genotypes to prevent the further acquisition of different vaccine preventable HPV genotypes, as recommended in previous study ${ }^{16}$. Another associated risk factor for abnormal cytology was HIV infection, although our participants with HIV-infection are all well-controlled by anti-retroviral therapy (ART). However, it is not clear from the present study of the cross-sectional design whether HIV causes impaired clearance of the HPV infection, or HIV-infected individuals reacquire HPV more frequently.

Table 2

Multiple logistic regression analysis to determine the factors associated with abnormal anal cytology

\begin{tabular}{|c|c|c|c|c|}
\hline \multirow[t]{2}{*}{ HPV positive individuals ( $n=377$ ) } & \multicolumn{2}{|c|}{ Univariate analysis } & \multicolumn{2}{|c|}{ Multivariate analysis } \\
\hline & OR $[95 \% \mathrm{Cl}]$ & $\begin{array}{l}P \\
\text { value }\end{array}$ & OR $[95 \% \mathrm{Cl}]$ & $P$ value \\
\hline Age $(+1$ year $)$ & $1.03[1.01-1.06]$ & .008 & $1.03[.99-1.06]$ & .094 \\
\hline HIV infection & $\begin{array}{l}12.06[2.90- \\
50.18]\end{array}$ & .001 & $6.52[1.52-28.46]$ & .012 \\
\hline $\begin{array}{l}\text { Number of detected hr-HPV } \\
\text { genotypes }\end{array}$ & $1.65[1.38-1.97]$ & $<.001$ & $1.57[1.30-1.90]$ & $<.001$ \\
\hline $100 \%$ use of condom & $1.35[.67-2.72]$ & .395 & & \\
\hline No. of lifetime sexual partner & $2.45[1.00-5.99]$ & .051 & & \\
\hline Past treatment history of STI & $1.62[.77-3.41]$ & .203 & & \\
\hline
\end{tabular}

There are some limitations to be considered in the present study. First, this was a cross-sectional study, and it is difficult to assess whether the HPV infection has been a transient infection or a persistent one, or a re-infection. Second, this study only analyzed hr-HPV genotypes, therefore not all genotypes were assessed. Third, cytology results for HIV-uninfected subjects were reported only using pap smear, then translated to Bethesda system using previously reported relationship of the two systems ${ }^{12}$. 
In conclusion, we found that hr-HPV, especially 9 valent HPV vaccine preventable HPV, was highly prevalent among MSM in Japan. Also, it was shown that increased number of hr-HPV genotypes were strongly associated with anorectal pre-cancer. Universal HPV vaccination for Japanese MSM should be considered to prevent HPV related malignancy, although further studies is needed for assessing health impact and cost-effectiveness of nine-valent HPV vaccination.

\section{Methods}

\section{Ethics statement}

A written informed consent for HPV and anal cytology testing was obtained from all participants. This study was approved by the ethics committee of the National Center for Global Health and Medicine (approval no. NCGM-G-002390). This study was implemented in accordance with the provisions of the Declaration of Helsinki.

\section{Study population}

We have an existing cohort of HIV-infected MSM, who are on treatment at AIDS Clinical Center (ACC), National Center for Global Health and Medicine (NCGM), Tokyo, as well as a non-HIV-infected MSM cohort at the Sexual Health Clinic (SHC) outpatient in the same center. We conducted a cross-sectional study in these two groups between January 1,2019, and August 31, 2019. Eligibility criteria included male sex, age $\geq 16$ years from SHC and age $\geq 20$ years from ACC, Self-reported history of having sex with men, willingness to undergo anal swab sampling to test for HPV infection, HPV genotypes (GTs), and anal cytology.

\section{Data collection}

Each participant completed a standardized questionnaire. The information gathered included sociodemographic data (age), sexual behavior data (number of lifetime sexual partners, number of sexual partners in the last 6 months, use of condom, and history of sexually transmitted diseases).

\section{HPV Detection and Cytology}

Samples were collected using anal canal swab, and all samples were taken and processed at the external laboratory, LSI Medience Corporation Tokyo, Japan. HPV DNA was detected by Hybrid Capture-based methodology described previously ${ }^{10}$. This included only oncogenic or hr-HPV genotypes $(16,18,31,33$, $35,39,45,51,52,56,58,59$, and 68$)^{11}$. For cytology, a liquid based cytology was prepared for routine Pap staining; cytology results were reported first using the Papanicolaou Classification (Class I-V) ${ }^{13}$, and also reported using the modification of the Bethesda System classification for cervical cytology: categories NILM (negative for intraepithelial lesion or malignancy), ASC-US (atypical squamous cell of undetermined significance), LSIL (low-grade squamous intraepithelial lesion), ASC-H (atypical squamous cells, cannot exclude high-grade lesion) and HSIL (high-grade squamous intraepithelial lesions) ${ }^{13}$. Cytology results for HIV-infected subjects were reported using both systems, but results for HIV-uninfected subjects were 
reported only using pap smear, then translated to Bethesda system using previously reported relationship of the two systems ${ }^{12}$.

\section{Statistical Analysis}

All statistical tests were evaluated at a .05 level of significance. Statistical analyses were performed with GraphPad Prism 7.0, and regression analysis was performed by Statistical Package for Social Sciences (SPSS) software, version 23.0. We reported categorical data as number (n) and percentage (\%), while continuous variables were reported as median and interquartile range (IQR). We compared differences in sociodemographic, and sexual behaviors between HIV-infected and uninfected subjects and performed descriptive analysis. Mann-Whitney $U$ tests were used for continuous variables, and Chi-squared/Fisher's exact tests were used for categorical variables. Odds ratio (OR) of significant factors associated with HPV infection and development of anal pre-cancerous lesions were calculated using logistic regression analysis. Multivariate logistic regression models were then applied to significant factors found in univariate analysis.

\section{Declarations}

\section{Acknowledgements}

The authors thank all staff members of AIDS Clinical Center, National Center for Global health and Medicine, Tokyo, Japan, for their contributions in this study.

\section{Author information}

\section{Affiliations}

AIDS Clinical Center, National Center for Global Health and Medicine, Tokyo, Japan.

\section{Contributions}

Conceived and designed the experiments: D.S., D.M., M.T. Performed the experiments: D.S., D.M., K.W., N.A., H.U., Y.Y., A.T., J.T., K.T., K.T., Y.K. Analyzed the data: D.S., D.M., K.W., H.G., S.O. Supervised the study and manuscript drafting: D.M., K.W., H.G., S.O. Wrote the paper: D.S. All of the authors critically revised this manuscript for important intellectual content and approved the final version submitted for publication.

\section{Corresponding author}

Correspondence to Daisuke Shiojiri,

\section{Additional Information}

\section{Conflict of interests}


The authors declare no conflict of interests regarding the present study.

\section{Funding}

This study was supported in part by funds from the National Center for Global Health and Medicine [grant no. 19A2011]

\section{References}

1. Vuyst, D. H., Clifford, G. M., Nascimento, M. C., Madeleine, M. M. \& Franceschi, S. Prevalence and type distribution of human papillomavirus in carcinoma and intraepithelial neoplasia of the vulva, vagina and anus: a meta-analysis. Int. J. cancer. 124, 1626-1636 (2009).

2. Daling, J. R. et al. Human papillomavirus, smoking, and sexual practices in the etiology of anal cancer. Cancer. 101, 270-280 (2004).

3. D'Souza, G. et al. Incidence and epidemiology of anal cancer in the multicenter AIDS cohort study. J. Acquir. Immune Defic. Syndr. 48, 491-499 (2008).

4. Phanuphak, N. et al. Anal human papillomavirus infection among Thai men who have sex with men with and without HIV infection: prevalence, incidence, and persistence. J. Acquir. Immune Defic. Syndr. 63, 472-479 (2013).

5 . Ferlay, J. et al. Cancer incidence and mortality worldwide: Sources, methods and major patterns in GLOBOCAN 2012. Int. J. Cancer. 136, E359-86 (2015).

6. Park, I. U. \& Palefsky, J. M. Evaluation and management of anal intraepithelial neoplasia in HIVnegative and HIV-positive men who have sex with men. Curr. Infect. Dis. Rep. 12, 126-133 (2010).

7. Meites, E. et al. Human papillomavirus vaccination for adults: updated recommendations of the Advisory Committee on Immunization Practices. MMWR Morb. Mortal. Wkly. Rep. 68, 698-702 (2019).

8. Food and Drug Administration. Prescribing information [package insert]. Gardasil 9 (human papillomavirus 9-valent vaccine, recombinant). Silver Spring, MD: US Department of Health and Human Services, Food and Drug Administration. https://www.fda.gov/media/90064/download. (2018).

9. Nagata, N. et al. Prevalence of Anal Human Papillomavirus Infection and Risk Factors among HIVpositive Patients in Tokyo, Japan. PLoS One. 10 (9), e0137434 (2015).

10. Yoshikawa, H. et al. Detection and typing of multiple genital human papillomaviruses by DNA amplification with consensus primers. Jpn. J. Cancer Res. 82, 524-531 (1991).

11. IARC Working Group on the Evaluation. of Carcinogenic Risks to Humans Human papillomaviruses. World Health Organization. 90, 1-636 (2007).

12. van Hamont, D., van Ham, M. A., Bakkers, J. M., Massuger, L. F. \& Melchers, W. J. Evaluation of the SPF10-INNO LiPA human papillomavirus (HPV) genotyping test and the Roche Linear Array HPV genotyping test. J. Clin. Microbiol. 44, 3122-3129 (2006). 
13. Hugh, M., Roman, L., William, W. \& Robert, A. The current status of the Papanicolaou smear. CA. cancer J. Clin. 45, 305-320 (1995).

14. Ping-Feng, W. et al. Anal human papillomavirus and its associations with abnormal anal cytology among men who have sex with men. Sci. Rep. 10, 3165 https://doi.org/10.1038/s41598-020-59967-4 (2020).

15. Li, X. et al. Anal HPV/HIV co-infection among Men Who Have Sex with Men: a cross-sectional survey from three cities in China. Sci. Rep. 6, 21368 (2016).

16. King, E. M. et al. Human papillomavirus DNA in men who have sex with men: type-specific prevalence, risk factors and implications for vaccination strategies. Br. J. Cancer. 112, 1585-1593 (2015).

\section{Figures}




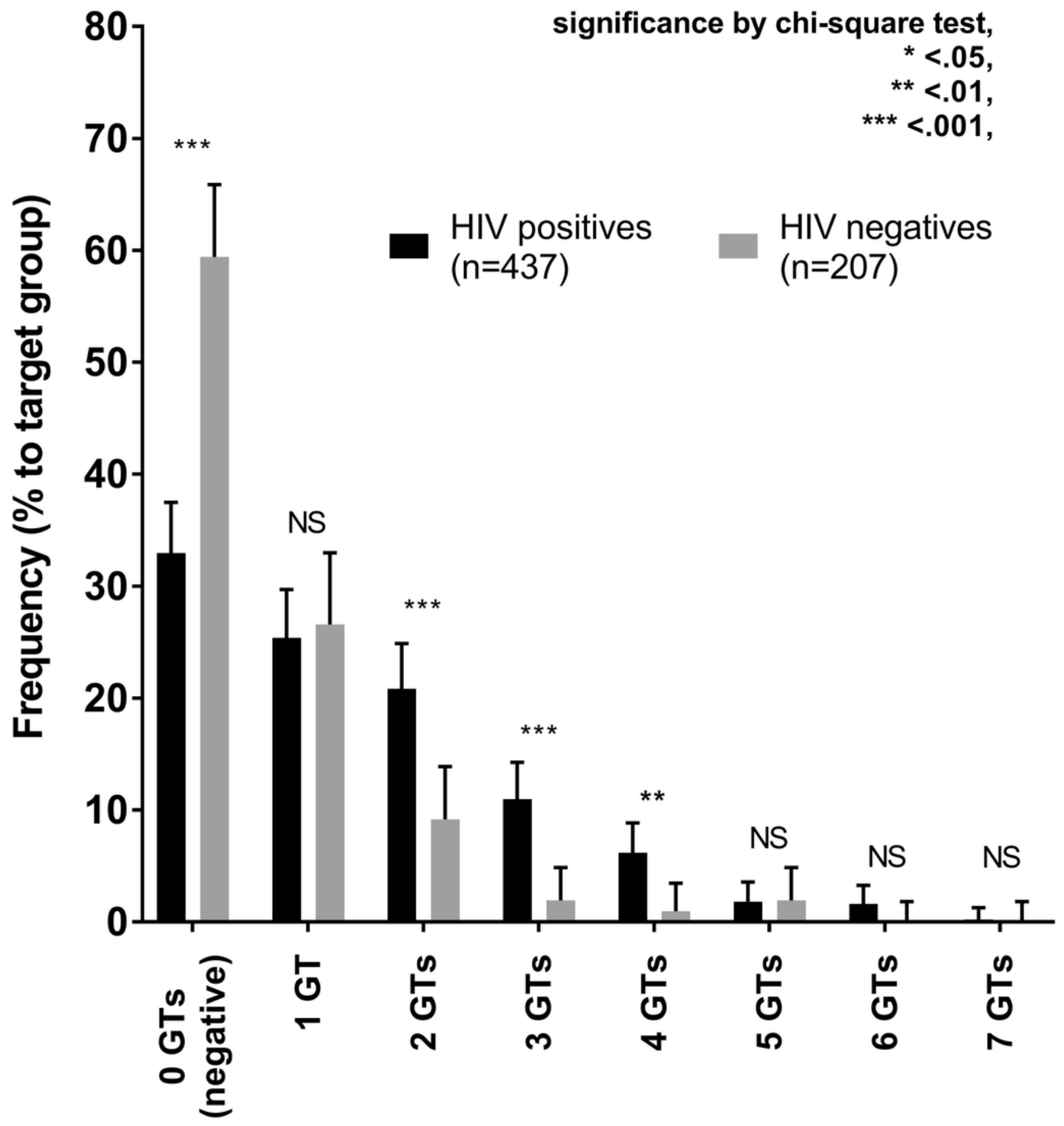

Figure 1

Number of hr-HPV genotypes in HIV-infected and uninfected MSM. Frequency of number of genotypes identified between HIV-infected and uninfected subjects were compared. Abbreviations: GT, genotype, hrHPV, high-risk types of human papillomavirus. 
a

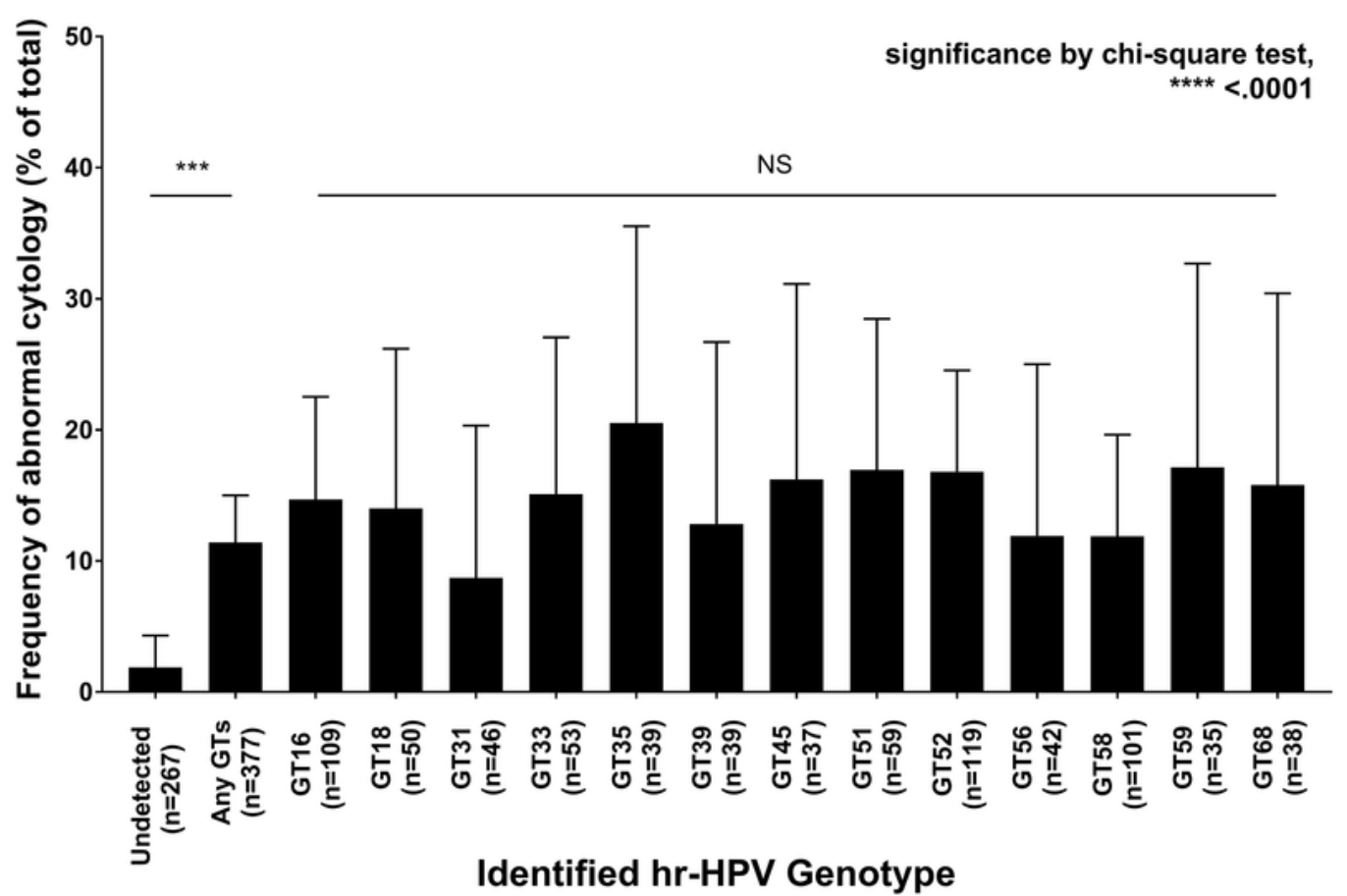

b

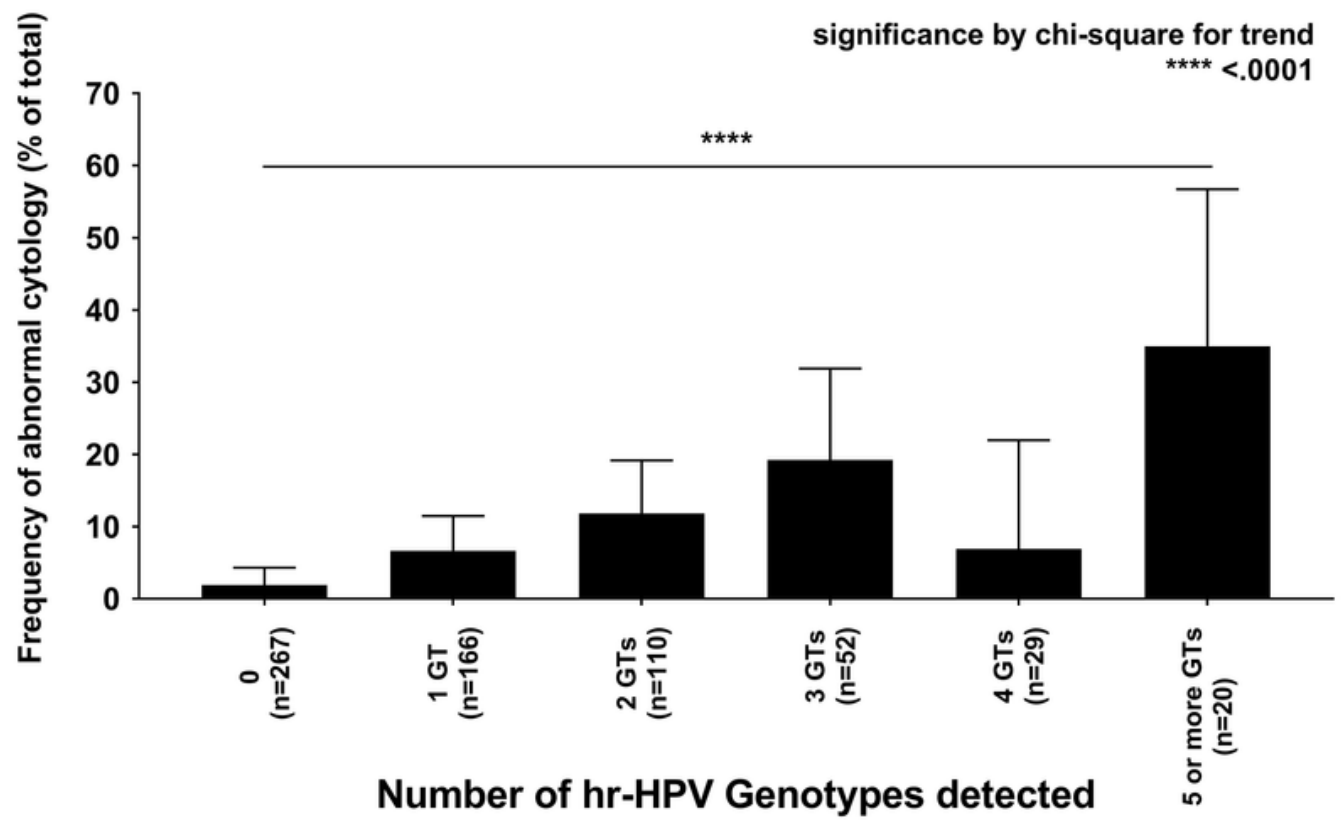

Figure 2

Frequency of abnormal anal cytology of anorectal swab. a) Frequency of abnormal anal cytology results (LSIL, ASC-H, or HSIL) was presented in accordance with the hr-HPV genotypes identified in all the subjects (644). b) Frequency of abnormal cytology results was calculated in accordance with the number of hr-HPV genotypes. Abbreviations: GT, genotype; hr-HPV, high-risk types of human papillomavirus. 


\section{Supplementary Files}

This is a list of supplementary files associated with this preprint. Click to download.

- DaisukeShiojiriSupplementaryFiguresS123AnalHPVamongMSMversion1.pptx

- DaisukeShiojiriSupplementaryTableS1AnalHPVamongMSMversion2.docx 